\title{
ESTUDIANTES RURALES AGRÓNOMAS EN UN CAMPO PROFESIONAL MASCULINIZADO
}

\section{WOMEN RURAL AGRONOMIC STUDENTS IN A MASCULINIZED PROFESSIONAL FIELD}

Jorge García Villanueva*, José Zenón Escobar Pérez**, Claudia Ivonne Hernández Ramírez Universidad Pedagógica Nacional*, Dirección General de Educación Tecnológica Agropecuaria**, México.

Correspondencia: jvillanueva@upn.mx

\section{RESUMEN}

El objetivo de esta investigación fue analizar las expectativas académicas, profesionales y laborales de cinco mujeres estudiantes de la carrera de ingeniería en agronomía con edades entre los 21 a 23 años. La metodología utilizada fue cualitativa, con un enfoque interpretativo, el instrumento fue un guión de entrevista y la técnica fue una entrevista semiestructurada. Los hallazgos indican que aún prevalece una forma de pensar que considera que las carreras de corte agropecuario están reservadas para los hombres; sin embargo, el desempeño académico y actitudes de las jóvenes demostraron una participación constante y sobresaliente comparada con la de sus compañeros; incluso la matrícula escolar en instituciones de educación superior agrícola del centro del país muestra signos de simetría.

Palabras clave: educación superior, ingeniería, expectativas académicas, estudiantes agrónomas.

\section{ABSTRACT}

The objective of this research was to analyze the academic, professional and labor expectations of five female students of the agronomy engineering career, aged 21 to 23 . The methodology used was qualitative, with an interpretative approach, the instrument was an interview script and the technique was a semi-structured interview. The findings indicate that a way of thinking still prevails that considers that agricultural careers are reserved for men; however, the academic performance and attitudes of the young women showed constant and 
outstanding participation compared to their peers; even school enrollment in agricultural higher education institutions in the center of the country shows signs of symmetry.

Key words: Higher education, engineering, academic expectations, agronomic students.

\section{INTRODUCCIÓN}

El presente espacio está enfocado en mostrar una visión general de cómo se viven las estudiantes rurales en un campo profesional masculinizado, se exponen los principales argumentos desde una perspectiva de género para comprender cuál ha sido la experiencia, expectativas e intereses de un grupo de mujeres en un ámbito considerado exclusivamente para hombres.

Desde la perspectiva de Bonder (1994), en la década de los 90 del siglo XX, los países de América Latina se vieron inmersos en reformas institucionales dirigidas a lograr un sistema que atendiera la agenda local y global; en la cuestión educativa era necesario un análisis profundo sobre los contenidos, las metodologías de enseñanza y evaluación, debido a los nuevos problemas de las sociedades contemporáneas como la igualdad de oportunidades para las mujeres en y desde la educación.

De acuerdo con lo anterior, se exponen datos de las mujeres mexicanas, partiendo de la visión tradicional que se ha tenido de ellas en el ámbito rural, su participación en la economía del país, su inclusión en la educación y su preferencia por matricularse en el nivel superior en carreras relacionadas por las ciencias sociales, humanidades y ciencias de la salud; en estos dos últimos rubros se ilustran sus particularidades que enfrentan al acceder a la educación superior, al mercado laboral y las posibilidades de trascendencia profesional y económica.

En este sentido, también se presentan cifras de mujeres que estudian agronomía en instituciones de educación superior agrícola y en escuelas de la zona metropolitana de la Ciudad de México, la proporción de inscritos en esta carrera casi muestran simetría; como en el caso de la Universidad Autónoma Agraria Antonio Narro (UAAAN) en sus campus ubicados en Saltillo, Torreón, Coahuila en el Norte del país y el centro donde se ubica el Instituto Tecnológico de Roque en Guanajuato, México.

Incluso las reformas a leyes y normas se efectuaron con el propósito de garantizar una mejor condición humana y promover la equidad de género. Asimismo, las políticas 
educativas reflejadas en los programas gubernamentales de mediano plazo buscan incorporar a un mayor número de mujeres a la vida social y productiva del país. El objetivo de la investigación es analizar las expectativas académicas, profesionales y laborales de cinco mujeres estudiantes de la carrera de ingeniería en agronomía en donde se recuperan sus experiencias y situaciones que vivieron en su paso por las aulas del Instituto Tecnológico Agropecuario de Roque en el estado de Guanajuato, México.

\section{LAAGRONOMÍA COMO UNA POSIBILIDAD DE ESTUDIO PARA LAS MUJERES RURALES}

En este apartado se presenta un panorama sucinto de la situación laboral y la distribución estadística de cómo se encuentra el ámbito de trabajo para las mujeres. Según el Instituto Nacional de Estadística Geografía e Informática (INEGI), en el 2010 se contabilizaron en México 112336538 habitantes, 57481307 eran mujeres y 54855231 hombres; lo que representaba en el rubro de la población femenina el $51.2 \%$. La población rural registrada en el mismo año fue de 26049128 habitantes lo que significó el 23.18\% del total de la población del país; en consecuencia, en el escenario rural el 50.4\% son mujeres.

En este contexto, una gran proporción son mujeres y todavía desempeñan el papel tradicional de esposa o cónyuge dependiente y los hombres se asumen como proveedores del hogar, ser jefa de familia en el medio rural comienza a incrementarse en comunidades donde se presentan altos índices de migración, viudez, separación de pareja o por trabajo mayormente remunerado por el esposo; asociado a tener derecho de propiedad sobre sus parcelas, participación directa en las labores del campo y contribuir con los gastos para el sostenimiento del hogar; también mantienen vivas las esperanzas de la resurrección y cambio generacional en el agro mexicano (CEDRSSA, 2014).

De las 13140 437, mujeres inmersas en el espacio rural, el 50.9\% participan activamente en la producción económica del país (INEGI, 2010), no obstante, en el mercado laboral se reproducen esquemas de segregación de actividades, inequidad de condiciones que impiden el acceso a otros incentivos de salud, mayor remuneración, seguridad social, educación, cultura, diversión y esparcimiento (CEDRSSA, 2014). Con base en la Encuesta Nacional de Ocupación y Empleo [ENOE] (INEGI, 2014), cuarto trimestre, la población rural femenina 3010470 labora en actividades de la rama agropecuaria el 18.1\%, industria manufacturera $18.6 \%$, comercio $24.3 \%$ y servicios diversos $16.1 \%$. 
El Programa Nacional para la Igualdad de Oportunidades y No Discriminación contra las Mujeres (PROIGUALDAD) 2013-2018 revela que las tasas de analfabetismo identifican a mujeres adultas mayores y mujeres indígenas con mayores rezagos $28.7 \%$ y $35.1 \%$ en ese orden. Dicho programa también alude a la tasa de asistencia escolar de niñas que están por cursar la educación primaria y secundaria, esto es el 96.4 y $86.4 \%$, respectivamente. No obstante, en comunidades rurales el $6.6 \%$ de niñas entre 6 y 14 años no acuden a la escuela (Gobierno Federal, 2013a).

Los datos sobre los indicadores de suficiencia educativa demuestran que, desde la educación primaria hasta la educación media superior, las mujeres presentan un aprovechamiento elevado en comparación con sus pares los varones, sin embargo, los hombres tienen mayores posibilidades de continuar con sus estudios superiores. Según PROIGUALDAD 2013-2018, en la educación media superior se registró que el 56.5\% de las mujeres entre los 16 y 18 años asiste a este nivel educativo y $23.7 \%$ de las mujeres entre los 19 y 24 años acude a la educación superior (Gobierno Federal, 2013b).

De acuerdo con la Asociación Nacional de Universidades e Instituciones de Educación Superior (ANUIES, 1999; 2012), las mujeres prefieren matricularse en educación y humanidades el 68.4\% y en ciencias de la salud el $64.7 \%$, y existe poca presencia de éstas en las ingenierías, participan tan sólo un 31.1\% y 35.4\% en las ciencias agropecuarias. En cuanto a las mujeres pertenecientes al Sistema Nacional de Investigadores (SNI) se estima un $34.1 \%$. Las universidades más importantes que ofrecen la carrera de agronomía en México presentan la siguiente situación en década y media del siglo XXI (ver tabla 1): 
Tabla 1

Instituciones de Educación Superior Agrícola más importantes en México

\begin{tabular}{|c|c|c|c|}
\hline Institución & Mujeres & Hombres & Total \\
\hline UAAAN $^{1}$ & 1242 & 3933 & 5175 \\
\hline $\mathrm{UACh}^{2}$ & 1769 & 2660 & 4329 \\
\hline UAEMéx ${ }^{3}$ & 257 & 461 & 718 \\
\hline $\mathrm{UAM}^{4}$ & 353 & 355 & 708 \\
\hline ITR $^{5}$ & 140 & 381 & 521 \\
\hline UNAM FES-C ${ }^{6}$ & 117 & 145 & 262 \\
\hline
\end{tabular}

Elaboración propia (2018)

En la Ciudad de México y su área conurbada la carrera de agronomía tiende a establecer paridad en la representación numérica, las mujeres acuden a estudiar en las instituciones como la Universidad Autónoma Metropolitana en Xochimilco el 49.8\%, la Facultad de Estudios Superiores Cuautitlán de la Universidad Nacional Autónoma de México el 44.6\% y en la Universidad Autónoma de Chapingo el 40.8\%. Sin embargo, en la universidad Antonio Narro se registra el $24 \%$ y en el Instituto Tecnológico con el $27 \%$ en su matrícula está representada por las mujeres. En México las jóvenes tienden a inscribirse en programas no agrícolas como educación, comunicación, ciencias de la salud, ciencias sociales y administrativas (Vázquez García y Zapata Martelo, 2005; Bustos Romero, 2012).

\section{LA PERSPECTIVA DE GÉNERO DESDE LAS POLÍTICAS PÚBLICAS EN MÉXICO}

Desde este panorama se describe el papel de la perspectiva de género desde las políticas públicas en México y se resalta la importancia de su incursión, los cambios y mejoras en programas para propulsar la igualdad de oportunidades entre las personas. En la década de los setenta, en México, se efectuaron distintas reformas al artículo $4^{\circ}$ constitucional, a los códigos civiles y penales, resultado de movimientos sociales en pro de los derechos de

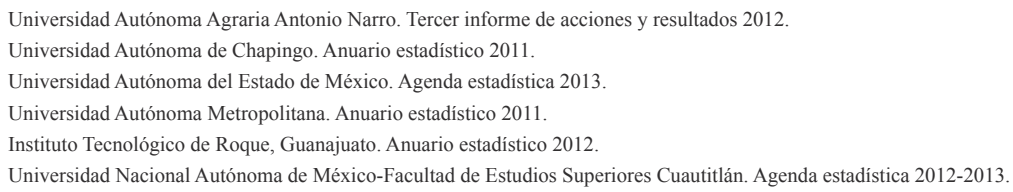


las mujeres, en diferentes entidades de la República Mexicana (INMUJERES, 2014). Al inicio de la década de los ochenta, se estableció el Programa Nacional de Integración de la Mujer; además en 1985 se crea la Comisión para coordinar las actividades y los proyectos sectoriales relacionados con el mejoramiento de la condición social de las mujeres, producto de la participación de México en la Tercera Conferencia Mundial sobre la Mujer en Nairobi, 1985 (INMUJERES, 2014).

El Plan Nacional de Desarrollo (PND) 1995-2000 proponía corregir las desigualdades en las oportunidades educativas y de empleo entre hombres y mujeres (Gobierno Federal, 1995), erradicar la violencia en sus diversas manifestaciones, apostándole a reformas de leyes para la defensa de sus derechos y pleno ejercicio de la ciudadanía. Lo anterior para dar cumplimiento a los compromisos de la Declaración de Beijing y su Plataforma de Acción aprobada en la IV Conferencia Mundial sobre la Mujer (ONU, 1995), en la que se reconocía la necesidad de que la mujer tuviera acceso pleno y condiciones de igualdad de educación y de capacitación como una de las doce esferas de preocupación que deberían proveer y vigilar los gobiernos y la comunidad internacional; durante este periodo se formula el Programa Nacional de la Mujer, para coordinar el cumplimiento de las políticas en el tema (INMUJERES, 2014).

En el periodo del 2001-2006, con el arribo del Partido Acción Nacional (PAN) después de 71 años de hegemonía del Partido Revolucionario Institucional (PRI), se establecieron una serie de acciones para enfrentar retos relacionados con la vulnerabilidad, la pobreza, la salud, el empleo, la educación y el liderazgo en sus respectivos contextos. En la agenda social se estableció el Programa para Jefas de Familia, en materia económica se impulsaron los Programas de Desarrollo Productivo de la Mujer y de la Mujer Campesina. En cuanto al rubro educativo se promovieron programas y acciones con énfasis en la equidad y la cobertura en la formación de las mujeres; como también en materia de gobierno, se publica la Ley del Instituto Nacional de las Mujeres, se sentaron las bases para la creación del Instituto Nacional de la Mujer y se pusieron en marcha el Programa Nacional para la Igualdad de Oportunidades y No Discriminación contra las Mujeres (Gobierno Federal, 2001).

En el PND 2007-2012; se planteó como un eje de política: la igualdad de oportunidades y como principal estrategia generar políticas públicas transversales con perspectiva de género en toda la Administración Pública Federal, con la finalidad de promover la transversalidad en los gobiernos estatales y municipales. Destacando acciones como el Sistema Nacional para la Igualdad entre hombres y mujeres, el Programa 
de Fortalecimiento a la Transversalidad de la Perspectiva de Género. Para el eje de transformación educativa figuraron los programas: Becas para Madres Jóvenes y Jóvenes Embarazadas y de Desarrollo Humano Oportunidades (Gobierno Federal, 2007).

Con el retorno del PRI, después de 12 años del gobierno de derecha, se planteó en el PND 20132018, incorporar la perspectiva de igualdad de género en las políticas públicas, programas, proyectos e instrumentos compensatorios como acciones afirmativas de la Administración Pública Federal y se publicó el Programa Nacional para la Igualdad de Oportunidades y no Discriminación contra las Mujeres 2013-2018 (Gobierno Federal, 2013a).

La revisión de los planes sexenales y sus criterios de orientación de las normas, leyes y políticas se imbricaron con la posibilidad del reconocimiento a los derechos de las mujeres en México; garantizar la igualdad de oportunidades para alcanzar su pleno desarrollo y trascendencia en todas las áreas de la vida social y productiva del país. Por lo que a partir del enfoque de estudio de las políticas desde la planeación gubernamental y la orientación sectorial; es posible sistematizar la información mediante la elaboración de diagnósticos, evaluación y escenarios prospectivos que recuperen las voces de los directamente involucrados (Miranda López, 2004).

\section{Método}

La presente investigación se abordó desde un enfoque cualitativo, porque refiere la realidad del contexto natural, es decir, del objeto de estudio, con base en sus cualidades y la descripción de sus características. El estudio fue interpretativo (Hernández Sampieri, Fernández Collado y Baptista Lucio, 2004) porque permitió especificar los perfiles de las jóvenes involucradas, describir las situaciones pues era necesario comprender el mundo y la vida de las estudiantes de la carrera de agronomía (Kvale, 2011).

La estrategia de selección de participantes fue por el criterio de conveniencia y la técnica seleccionada fue la de bola de nieve, la cual consistió en localizar a algunas jóvenes con disposición, conocimiento y experiencia sobre el tema, hasta sumar cinco estudiantes que no llevaran una relación estrecha, simplemente, conocidas de la licenciatura en agronomía (Ito Sugiyama y Vargas Núñez, 2005).

El instrumento para recolectar la información fue un guión de entrevista; para orientar el hilo discursivo de forma semiestructurada (Flick, 2004), que facilitó el intercambio 
de información sobre temas abiertos como la elección de carrera, su proceso formativo, posibilidades de empleo y proyecto de vida.

La recolección, análisis e interpretación de datos se llevó a cabo mediante la discriminación anticipada de las categorías de análisis: expectativa académica, expectativa profesional y proyecto de vida (Bonilla Castro y Rodríguez Sehk, 2005). Se emplearon seudónimos con la finalidad de reservar la confidencialidad de las participantes. El informe final se realizó a partir del resumen de las respuestas de las entrevistadas en textos breves; fortaleciendo sus palabras en marcos referenciales y conceptuales que dieron cuenta de un estudio más amplio.

\section{ANÁLISIS DE LOS RESULTADOS}

En esta sección se presentan los principales hallazgos y el análisis de resultados de la investigación:

Tabla 2: Características de las mujeres entrevistadas

\begin{tabular}{|c|c|c|c|c|c|c|}
\hline Seudónimo & Edad & Localidad & $\begin{array}{c}\text { Promedio } \\
\text { escolar }\end{array}$ & $\begin{array}{c}\text { Estado } \\
\text { civil }\end{array}$ & Hijos & Trabaja \\
\hline Grecia & 21 años & Rural & 9.2 & Soltera & No & $\mathrm{Si}$ \\
\hline Nuria & 22 años & Urbana & 9.0 & Soltera & No & $\mathrm{Si}$ \\
\hline Laura & 21 años & Urbana & 8.8 & Soltera & No & No \\
\hline Ana & 23 años & Rural & 9.4 & Soltera & No & Si \\
\hline Martha & 22 años & Urbana & 9.0 & Soltera & No & No \\
\hline
\end{tabular}

\section{EXPECTATIVA ACADÉMICA}

Las jóvenes entrevistadas dieron cuenta de que el proceso de elección de escuela y carrera se dio por la situación económica, cercanía y preferencia por la misma:

\section{GRECIA}

“...bueno, principalmente, porque, la economía, o sea eh, con lo que cuento yo, pues, me alcanzaba para entrar a esta universidad, al principio yo no quería estudiar esto, por, bueno yo quería estudiar medicina, pero por la economía -no me alcanzaba- pues yo me pago mis estudios". 


\section{NURIA}

"Pues agronomía fue mi tercera opción de carrera, primeramente quería estudiar lo que es gastronomía o en este caso, para educadora... pues eran carreras que demandaban demasiado dinero... pues no, no tenía para cubrir esos gastos".

\section{ANA}

"Este eh, bueno la agronomía es una carrera... Que me llama mucho la atención, tenía, tiene buen prestigio aquí la escuela, aparte también por la ubicación es la que más acceso tengo".

\section{MARTHA}

"Más que nada fue porque... mis papás... toda mi familia si va a venir a aquí a Celaya... y dije, bueno pues, Roque está bien... investigué en internet, me empapé primero de la información, que ofrecía la escuela, dije... me voy a agronomía”.

Como se ilustra en los fragmentos anteriores, la situación económica fue un factor fundamental para la elección de la escuela y carrera, en el caso de Grecia, se puede apreciar su indecisión en cuanto a qué estudiar y la opción elegida se ajustó a sus posibilidades; por su parte, Nuria también enfatiza que el dinero se presentó como el impedimento fundamental para que ella eligiera una carrera diferente.

Para Ana, la ubicación y el prestigio de la escuela fueron suficientes criterios para que ella estudiara, la decisión de Martha estuvo permeada por el apoyo de su familia y la somera indagación que realizó de la institución para poder estudiar en la misma.

Se observa que dichas decisiones se encuentran sumergidas en una realidad cercana para las participantes en relación con el acceso a la educación superior, ubicada en la posición económica de la cual provienen, la falta de orientación dirigida a la elección de una carrera que permitiera identificar el perfil de ingreso, egreso, las oportunidades laborales y expectativas personales; aspectos fundamentales para efectuar una elección pertinente, segura y consciente.

\section{GRECIA}

"Pues ellos me dijeron que le echara ganas y que si sentía que yo podía pues me metiera pero nada más a eso, económicamente no contaba con ellos". 
Aún proviniendo de la misma escuela, los casos varían por distintas circunstancias, un ejemplo es el de Grecia, su familia apoyaba la decisión de estudiar, sin embargo, no contaba con el sustento económico, ella tenía que solventar su carrera.

De acuerdo con los resultados de su investigación, Licea de Arenas, Sandoval y Arenas (2003) mencionan que las condiciones educativas de las mujeres han experimentado cambios en las primeras décadas del siglo XIX, de las casi 60,000 mujeres que habitaban la Ciudad de México en 1810, sólo llegaban a 300 las bien educadas. Hoy en día, las estadísticas revelan que el porcentaje de mujeres con licenciatura está aumentando rápidamente. Sin embargo, al haber desigualdad en términos de estudios de doctorado en el extranjero, puestos y distinciones, se asume que su inserción al proceso de producción de conocimiento ha sido lento y que han tenido que enfrentarse a la ignorancia, discriminación o ninguneo lo cual podría haber minado su confianza y afectado su destino.

\title{
EXPECTATIVA PROFESIONAL
}

Se encontró que a diferencia de los hombres que estudian en el instituto, las estudiantes enfrentan mayor exigencia que repercute en un gran esfuerzo relacionado con su formación académica.

\section{MARTHA}

"Este eh... bueno en algunas ocasiones es que hay maestros, faltan bas...tante (con temor) y otra es que, este, hay muchos maestros que, si cargan mucho la mano, si dejan mucho trabajo..."

\begin{abstract}
ANA
"Ahorita... ahorita por ejemplo traemos alguna diferencia con un profesor y nos hemos topado con la limitación de que no nos están apoyando en algunas oficinas... Eh... es que el profesor es muy inaccesible, nos pone trabajos... trabajos que son inequitativos, entonces él está inflexible y pues se está valiendo desde su posición como maestro..."
\end{abstract}




\section{NURIA}

"Pues hay maestros que si lo decían de plano que una mujer no tenía nada que hacer aquí... En primer semestre, un maestro, un doctor justamente; mencionó que una mujer no servía para esto".

\section{ANA}

“...quizá en algún momento de la carrera nos topamos con algún profesor que era machista, pero pues de ahí no paso".

\section{LAURA}

"La actividad por la tarde es pues muy... muy tranquila como que no hay mucha gente en la escuela, esta así solitaria”.

\section{NURIA}

“...pues yo no puedo ir caminando porque ya te robaron el teléfono, te robaron cualquier cosa y son chavitos".

La visión patriarcal aún prevalece en la ideología de las personas, al seguir predicando que existe de forma tajante una división sexual en las carreras. En el caso de Nuria, el ejemplo se expone con el profesor que considera que las mujeres no son aptas para estar en la universidad.

De forma paralela, Martha y Ana señalan que el trato diferenciado en relación con el trabajo realizado en el aula, las actividades y tareas; dejan entrever que el profesor considera que la condición de mujeres las proyecta como carentes de inteligencia y la ingeniería agrónoma representa un reto difícil de superar porque es una carrera masculinizada, desde la perspectiva del docente.

Otro aspecto perturbador, es la violencia sufrida dentro del plantel debido a la inseguridad, situación que quebranta la integridad de las personas y desde una posición hegemónica, las mujeres son piezas clave para amenazar y asustar con facilidad. También, señalaron que algunos de sus compañeros comparten la visión del profesor, al seguir reforzando estereotipos sexistas. Sin embargo, la formación profesional debe descansar en la incorporación de mayores niveles de conocimiento, fomento del trabajo en equipo, 
capacidad de interacción simbólica, amplio conocimiento del proceso productivo, desarrollo de pensamiento innovador y anticipatorio y la construcción de mentalidades críticas y propositivas, sin importar el género de las personas. El desempeño profesional se alcanza cuando se desarrollan valores y actitudes dirigidos al beneficio de la sociedad, del entorno y de la persona, por lo tanto, el perfil de un estudiante de agronomía tendría que enfocarse en una actitud emprendedora, capacidad de adaptarse al cambio; considerar y respetar los factores culturales, ambientales y éticos involucrados en su trabajo, así como una capacidad de liderazgo (Córdova Duarte, Ramírez y Barbosa Jaramillo, 2011).

\section{GRECIA}

"Ah, pues de hecho aquí son muchos hombres, y como que, que nos echan tierra los muchachos, porque, porque, luego no admiten que una mujer puede ser mejor que los hombres; porque los hombres flojean más, no sé, o será que a mí me cuestan mis estudios por eso le echo muchas ganas".

"Sí, sí hay trabajo en equipo de hecho porque en nuestro género como que nos organizamos más, de hecho, luego organizamos a los hombres; aunque la carrera sea como que, de hombres, y pues ya estamos en pleno siglo XXI ya no es para que, esta carrera solo es de hombres..."

No obstante, se percibe que las jóvenes continúan manteniendo el compromiso y el profundo esfuerzo por trascender en sus expectativas profesionales, pese a que aún se siga pensando que las mujeres no tienen derecho a estudiar una profesión con una población mayoritariamente masculina. Al respecto, Gutiérrez Portillo y Duarte Godoy (s/f) mencionan que las identidades subjetivas de las estudiantes se ven influenciadas por la cultura y los discursos dominantes que reproducen la masculinización en una noción que matiza los roles femeninos y masculinos en las estudiantes.

\section{PROYECTO DE VIDA}

Las estudiantes tienen planes a corto y mediano plazo después de que hayan culminado la carrera, en los fragmentos discursivos se evocan experiencias que han tenido en el campo laboral y todavía se sigue manifestando el mandato heteronormativo de una forma sutil. 


\section{NURIA}

“...incluso hemos ido a varias empresas -en una- nos dijeron, que la mujer nada más para trabajar en campo, pues no están aptas para trabajar y otras cositas más... No pues fuimos a una visita, justamente...ellos nos dijeron aquí no dejamos entrar a mujeres..."

Nuria comenta su experiencia en una empresa de la región, se sorprende al escuchar la respuesta de la persona encargada de la misma, y tan sólo fue una visita.

Durante las prácticas agrícolas y viajes de campo, las jóvenes siempre trabajaron al parejo de sus compañeros, incluso recibieron su apoyo y reconocimiento, como también compartieron problemas comunes de la carrera:

\section{ANA}

"Pues yo me siento, eh capaz (con seguridad) y, pues en general en ambos sexos hay una limitante, se tiene la negativa de los productores que el agrónomo que va a enseñarles, pero si uno tiene la capacidad pues, es más fácil, además como le comentaba, el trabajo de campo no es el único de la agronomía, hay más".

Ana muestra seguridad en su argumento, al hacer mención que el campo no es la única opción para trabajar, existen otros ámbitos en los cuales se puede desempeñar de forma profesional.

De las jóvenes entrevistas, hay dos de ellas que trabajan en el periodo vacacional y comparten su experiencia, identificando aspectos que suelen obstaculizar las relaciones laborales.

\section{NURIA}

"Porque ahorita pues solo he trabajado como peón, pero si yo llego a un trabajo y yo como mujer a darles órdenes a hombres jornaleros es bien difícil que te hagan caso".

\section{GRECIA}

"Eh, ahorita no puedo trabajar porque, no sé, te absorben mucho tiempo las tareas; ahorita, y trabajo en vacaciones, siempre he trabajado en vacaciones desde que estaba en la prepa". 
ANA

"Me ayudo con la venta de tamales, los viernes vendo en el estacionamiento a mis compañeros de carrera... pero al parecer ya no quieren que venda algunas autoridades de la escuela".

\title{
GRECIA
}

"Me gustaría trabajar en el área de ecología -desarrollo sustentable-... me gustaría trabajar en la parte pública pero en la parte privada podría ser más adelante..."

\section{NURIA}

"Me gustaría especializarme en invernaderos pero sobre producción orgánica... Aunque... me gustaría trabajar en una dependencia tal vez SAGARPA o INIFAP”.

\section{LAURA}

“En zootecnia... Pues no se en algún rancho particular".

\begin{abstract}
ANA
“...en la transferencia de tecnología pero en los pequeños productores... Yo tengo la expectativa de trabajar en el INIFAP y CIMMyT".
\end{abstract}

\section{MARTHA}

"Ahorita me gustaría trabajar, en lo que viene siendo en lo agrícola, lo de la agricultura... me visualizaría trabajando en SAGARPA".

Las estudiantes, en su mayoría, prefieren laborar en la agricultura como promotoras de desarrollo agrícola y en el sector público. En cuanto a su proyecto de vida expusieron lo siguiente:

\section{GRECIA}

“...el inglés es algo esencial y para trabajar en algo más, que ganes más, necesitas tener más conocimientos una maestría un doctorado, porque quedarme así con una licenciatura siento como que no". 


\title{
LAURA
}

"Pues si se pudiera, terminando, un año después. Me gustaría seguir estudiando una maestría... en zootecnia...en Irapuato... ¿casarme después de terminar? Aun no sé...”

\begin{abstract}
ANA
“...ahorita mi prioridad es mi proyecto académico, terminar, terminar, mi carrera incorporarme, a la parte laboral, establecerme económicamente e independizarme de mis papás... Mmm pues casarme... creo que no es mi prioridad".
\end{abstract}

\section{NURIA}

“...si tengo planes, tengo planes de vida se lo que quiero hacer en dos años o lo que voy a estar haciendo... simplemente ahorita solo quiero trabajar para juntar para poder moverme para estudiar donde yo quiera estudiar mi maestría y no donde el destino me lo imponga. Me gustaría estudiar una maestría, pero en el caso de que no se pudiera por trabajos o por tiempos o porqué ya me casé pues si me gustaría tomar un diplomado también en invernaderos”.

En los discursos, de las mujeres estudiantes, aún prevalecen vestigios de la ideología androcéntrica, como el matrimonio, aunque no es un aspecto prioritario, Grecia y Laura, destacan la importancia de continuar estudiando y de alcanzar un grado académico más alto, una posición diferente en el ámbito laboral. En el caso de Laura, la posibilidad de casarse queda en la incertidumbre y Ana, busca la independencia económica y familiar.

Por su parte, Nuria tiene considerado un tiempo determinado para cumplir sus expectativas profesionales, laborales y sociales, no desea quedarse sólo con la preparación universitaria.

Durante siglos la presencia de las mujeres en la educación superior fue marginal y legitimada con argumentos sobre una supuesta inferioridad intelectual y la incapacidad para cumplir con la exigencia social de dedicarse a ser esposas y madres. Estos fueron obstáculos que limitaron su acceso a la educación universitaria. De acuerdo con Güereca Torres (2017) fue a partir de la década de 1970 que la aparición de las mujeres en la educación superior ha registrado un mayor aumento frente a la presencia masculina, sin embargo, ese crecimiento de matrícula no se ha extendido a todas las áreas del conocimiento ni a todos los niveles, lo cual tiene relación con situaciones etarias y socioeconómicas del entorno; así como con la prevalencia de referentes de género que asocian lo femenino y lo masculino con saberes diferenciados. 
Como se puede observar, la inclusión de mujeres en la educación superior es una realidad parcial porque todavía persisten diferencias en ciertas carreras, es decir, no en todas las licenciaturas las mujeres han logrado una igualdad en su participación. Aún existen profundos atavismos culturales que provienen de las estructuras familiares y los ámbitos escolares para que las mujeres no estudien carreras en ciertas disciplinas, pero también ocurre que en ciertas licenciaturas no se aprecia y se valora que los hombres lleven a cabo sus estudios profesionales (De Garay, Del Valle-Díaz-Muñoz, 2012).

\section{CONSIDERACIONES FINALES}

Al inicio del siglo, se dio apertura a nuevas conquistas en torno a la igualdad para las mujeres. En las décadas precedentes se han reconocido sus derechos como parte de los derechos humanos, en la medida que se exige la no discriminación por razones de género. El principio de igualdad legal ha generado importantes cambios para las mujeres y sus posibilidades, al menos teóricas, de acceder a las diferentes esferas sociales (Díez Gutiérrez, Terrón Bañuelos y Anguita, 2005).

Pese a las reformas constitucionales en algunos numerales, el enfoque de género continúa ausente en la legislación laboral. Las mujeres continúan en desventaja en todos los indicadores laborales y se enfrentan a discriminaciones abiertas o encubiertas, como los llamados "techos de cristal” en su ascenso a empleos jerárquicos (Espino, 2011).

Desde los estudios de género se devela que el pensamiento del ser humano es binario y que la diferencia sexual de los cuerpos es simbolizada por las culturas (Miguez Fernández, 2007). De ahí que en las distintas sociedades se han establecido prescripciones específicas y diferenciadas para las mujeres y los hombres, que varían dependiendo de la cultura, la geografía y la etapa histórica, lo que demuestra que el género está expuesto a cambios que imponen las condiciones sociales y por la invención de las personas.

Miguez Fernández (2007) señala que las construcciones simbólicas acerca de lo femenino y lo masculino suelen ser perdurables en el imaginario social y en las significaciones interiorizadas a nivel individual. Al analizar las expectativas académicas, profesionales y laborales de las mujeres participantes de la carrera de ingeniería en agronomía, se observó que se mantiene un código de género relacionado a las profesiones, puesto que los campos de estudio mantienen una división que denota espacios y relaciones de poder, que sancionan el paso de las personas e incluso pueden significar prohibición en el acceso, 
por razones de género. Como lo manifestaron en sus testimonios Martha, Ana y Nuria, en la categoría de expectativa profesional.

Estos pensamientos y esquemas culturales, basados en realidades sociales y económicas (García Villanueva y Hernández Ramírez, 2015), se infiltran de generación en generación moldeando expectativas distintas para mujeres y hombres que los ubica e identifica en ámbitos separados, intransferibles e inamovibles y limita fuertemente sus aspiraciones e intereses.

Al respecto, García Villanueva y Hernández Ramírez (2015) comentan que los estereotipos postran a las personas en un modelo fijo y general, que se rehúsa a prestar atención a las cualidades individuales; se trata de seguir manteniendo una imagen mental uniformada exageradamente simplificada, que transmite sin fundamento unas características que se atribuyen a un sexo invocando una supuesta diferencia natural.

Y en el mercado de trabajo se establece la discriminación entre los puestos laborales, de tal manera que existen ramas profesionales, tareas y oficios desarrollados exclusivamente para las mujeres y otros en donde sólo lo ejercen los hombres, como lo indicaron en sus argumentos Laura, Grecia, Ana y Nuria, desde su proyecto de vida.

Es importante destacar que, la mayoría de las jóvenes tuvieron que decidir su ingreso al Instituto Tecnológico Agropecuario por la situación económica y la cercanía a su lugar de origen, quizá de una forma planificada. Y la carrera de agronomía, no figuraba como una prioridad para estudiar.

Durante la entrevista, reconocieron que, a lo largo de la trayectoria escolar, mantuvieron mayor respeto, autonomía, responsabilidad, empatía, compromiso y su aprovechamiento fue sobresaliente, en comparación con sus compañeros. Además, manifestaron que algunos estudiantes se mostraban celosos de la presencia femenina y, por ello, el comportamiento que denotaban era de indiferencia y rechazo. Cuando tuvieron acercamientos al campo laboral a través de las prácticas profesionales, los trabajadores agrícolas mantenían renuencia al recibir órdenes por parte de una mujer.

En su proyecto de vida, figura el trabajar en el sector público como promotoras de desarrollo rural, extensionistas y asistentes técnicos en áreas como: agricultura sustentable, agricultura protegida, zootecnia e investigación. También consideran importante concluir la carrera, incorporarse al campo laboral para lograr autonomía económica y continuar su preparación 
con estudios de posgrado, algunas pretenden seguir con una especialidad, maestría o el doctorado. Cabe señalar que, la idea de conformar su propia familia todavía aparece, pero no como una prioridad en su vida; desean lograr sus metas académicas y profesionales, y después establecer un compromiso conyugal.

Desde las manifestaciones discursivas analizadas en la presente investigación, se observa que la imagen que tienen las estudiantes rurales, aún no se aleja de las labores mujeriles como: el matrimonio y la maternidad (Vázquez García y Zapata Martelo, 2005) a pesar de que se encuentran en el nivel superior y decidieron estudiar una carrera relacionada con la ingeniería, lugar no tan concurrido por mujeres; como en las ciencias sociales y humanidades, puesto que se sigue pensando que tienen exclusividad por género (Razo Godínez, 2008).

Además, la proporción de personas inscritas en el instituto y en la licenciatura de agronomía, no refleja simetría entre la población de mujeres y hombres; sin embargo, otras escuelas de nivel superior agrícola del centro del país han comenzado a mostrar en su matrícula simetría entre el alumnado. Quizá, este podría ser un indicador de que este ámbito de estudio no es exclusivo para los varones como tampoco las actitudes y desenvolvimiento que las jóvenes expresaron, puesto que sus aspiraciones académicas, profesionales y laborales se asemejan a las de sus compañeros (Emagister, 2014).

Las manifestaciones sexistas, actitudes discriminatorias y de exclusión se siguen presentando entre el alumnado y profesorado, puesto que consideran que el esfuerzo físico y mental sólo es para los hombres siguen considerando que las mujeres son delicadas y carecen de fuerza física y raciocinio (INFOAGRO, 2010).

Las estudiantes mencionaron que en el terreno práctico en ningún momento renunciaron a las extenuantes labores en las parcelas, ni a las tareas de dirección y toma de decisiones, sólo destacaron que los jornaleros solían ignorar sus instrucciones porque consideraban que éstas no representan figuras de autoridad y mando, por ser mujeres.

Habría que cuestionarse ¿cómo se llega a la igualdad de oportunidades para las personas? ¿cuál es el camino, se tendría qué construir o se seguiría el que ya está trazado? ¿los términos de igualdad y equidad se aplican para todas las personas sin importar el contexto de procedencia?

Al respecto, Martha Lamas (2003) argumenta que para que exista un desarrollo más equitativo y democrático del conjunto de la sociedad se requiere la eliminación de los tratos 
discriminatorios contra cualquier grupo; en el caso específico de las mujeres. Se requieren políticas públicas que consideren las condiciones culturales, económicas y sociopolíticas que coadyuvan con la discriminación hacia las mujeres.

Además, no basta con declarar la igualdad de trato, cuando en la realidad no existe igualdad sustantiva ni de oportunidades para todas las personas. Aún se sigue jerarquizando el papel que juegan los hombres en la familia y la sociedad. Es necesario cuestionar que sólo se tiene una convivencia de relaciones desiguales, que subsiste enmascarada en los discursos de igualdad, libertad y universalismo (Simón Rodríguez, 2006).

Desde la voz expuesta por las mujeres agrícolas, lo que se tendría que buscar es que la educación superior camine hacia la construcción de un modelo coeducativo que enfatice la igualdad entre los géneros, basado en el respeto a las diferencias y el reconocimiento de las personas, en donde se excluya toda práctica discriminatoria, sexista y misógina para evitar la fabricación y la reproducción de personas dóciles, sumisas, abnegadas y obedientes, llamadas mujeres.

Al respecto, en otros trabajos (García Villanueva y Hernández Ramírez, 2015) hemos considerado que es necesario realizar cambios estructurales en las instituciones, lo que implica comenzar a hacer uso de un lenguaje inclusivo, transformando el manejo de genéricos que en sí mismos excluyen a un sector de la población. Además de cuestionar la visión enajenante del modelo de la masculinidad hegemónica, brindando la oportunidad de deconstruir identidades femeninas y masculinas diferentes, libres de estereotipos y de una supuesta diferenciación natural construida con base en modelos sociopolíticos, culturales y religiosos que limitan fuertemente las aspiraciones e intereses de mujeres y hombres.

Como seres humanos, como sociedad evolucionada, civilizada tenemos que trabajar para desaparecer espacios profesionales masculinizados o feminizados pues ello contribuye a perpetuar los perniciosos e indeseables roles y estereotipos de género. En este sentido, conviene destacar el papel de las mujeres rurales como un elemento emancipador que permita transformar el sendero educativo de las carreras que están masculinizadas y dar paso a que otras mujeres deseen ubicarse en roles profesionales y laborales con mayor envergadura e impacto para la sociedad mexicana (Bonder, 1994). Además de ofrecer la posibilidad de otros modelos de ser mujeres con expectativas e intereses laborales con mayor estatus y rango e incursionar en ámbitos de mando y dirección que han sido espacios exclusivos para un determinado género. 


\section{REFERENCIAS}

I ANUIES (1999). Tipología de Instituciones de Educación Superior. México: ANUIES.

I ANUIES (2012). Anuario Estadístico 2013. México: ANUIES.

I Bonder, G. (1994). Mujer y educación en América Latina: hacia la igualdad de oportunidades. Revista Iberoamericana de Educación, 6, 9-48. https://doi.org/10.35362/rie601206

I Bonilla Castro, E. y Rodríguez Sehk, P. (2005). Más allá del dilema de los métodos. Colombia: Nomos.

I Bustos Romero, O. (2012). Mujeres en la educación superior la academia y la ciencia. Revista Académica de la Ciencia, 63(3), 24-33. Recuperado de: file:///C:/Users/jose.zenon/Downloads/Mujeres_Educ $\underline{\text { Superior.pdf }}$

I Centro de Estudios para el Desarrollo Rural Sustentable y la Soberanía Alimentaria [CEDRSSA] (2014). Cámara de Diputados. Condiciones Económicas y Sociales de las Mujeres Rurales en México. México.

I Córdova Duarte, G., Ramírez, L. V. y Barbosa Jaramillo, E. R. (2011). El perfil académico profesional del ingeniero agrónomo. Una propuesta renovada para el siglo XXI. Revista Latinoamericana de Estudios Educativos, 41(1-2), 143-178.

I De Garay, A. y Del Valle-Díaz-Muñoz, G. (2012). Una mirada a la presencia de las mujeres en la educación superior en México. Revista Iberoamericana de Educación Superior, 3(6), 3-30.

I Díez Gutiérrez, E., Terrón Bañuelos, E. y Anguita, R. (coords.). (2005). La cultura de género en las organizaciones escolares. Motivaciones y obstáculos para el acceso de las mujeres a los puestos de dirección. España: Octaedro.

I Emagister (2014). Las ingenierías dejan de ser cosas de hombres, ahora les toca el turno a ellas. Recuperado de: http://www.emagister.com/blog/crece-el-numero-de-mujeres-que-quieren-ser-ingenieras/

IEspino, A. (2011). Trabajo y género un viejo tema, ¿nuevas miradas? Nueva Sociedad, 232, 86-102.

I Flick, U. (2004). Introducción a la Investigación Cualitativa. España: Morata.

I García Villanueva, J. y Hernández Ramírez, C. I. (2015). La formación del profesorado de nivel preescolar: un campo que excluye a los hombres en la Ciudad de México. Revista Cientifica EntreCiencias: diálogos en la sociedad del conocimiento, Enes UNAM, http://www.entreciencias.enes.unam.mx/numero-7/\#.Vb $\underline{\mathrm{X} 8 \mathrm{YvnkUs}}$ 
I Gobierno Federal (1995). Plan Nacional de Desarrollo 1995-2000. Publicado en el Diario Oficial de la Federación 31 de mayo. México. Recuperado de: http://dof.gob.mx/nota detalle. php? codigo $=4874791 \&$ fecha $=31 / 05 / 1995$

\Gobierno Federal (2001). Plan Nacional de Desarrollo 2001-2006. Publicado en el Diario Oficial de la Federación 30 de mayo. México Recuperado de: http://dof.gob.mx/nota detalle. php?codigo $=766335 \&$ fecha $=30 / 05 / 2001$

\Gobierno Federal (2007). Plan Nacional de Desarrollo 2007-2012. Publicado en el Diario Oficial de la Federación 30 de mayo. México Recuperado de: http://dof.gob.mx/nota detalle. php? codigo $=4989401 \&$ fecha $=31 / 05 / 2007$

I Gobierno Federal (2013a). Programa Nacional para la Igualdad de Oportunidades y No Discriminación contra las Mujeres 2013-2018. Publicado en el Diario Oficial de la Federación 30 de agosto. México. Recuperado de: http:/www.inah.gob.mx/images/stories/Transparencia/2014/nodiscriminacion mujeres 2013_2018.pdf

I Gobierno Federal (2013b). Plan Nacional de Desarrollo 2013-2018. Publicado en el Diario Oficial de la Federación 20 de mayo. México. Recuperado de: http://dof.gob.mx/nota_detalle. php? codigo $=5299465 \&$ fecha $=20 / 05 / 2013$

I Güereca Torres, R. (2017). Violencia epistémica e individualización: tensiones y nudos para la igualdad de género en las IES. Reencuentro: Género y Educación Superior, 74, 11-32.

I Gutiérrez Portillo, S. y Duarte Godoy, M. (s/f). Ingenieras o ingenieros: cómo se conciben las mujeres en el campo de ingeniería. Recuperado de https:/www.oei.es/historico/congresoctg/memoria/pdf/Gutierrez.pdf

\Hernández Sampieri, R., Fernández Collado, C. y Baptista Lucio, P. (2004). Metodología de la Investigación. México: McGraw-Hill Interamericana.

I INEGI (2010). Censo de Población y Vivienda. Resultados Definitivos. Recuperado de: http://www3.inegi. org.mx/sistemas/temas/default.aspx?s=est\&c=17484

$\checkmark$ INEGI (2014). Encuesta Nacional de Ocupación y Empleo. Indicadores estratégicos. Cuarto trimestre. Indicadores estratégicos de ocupación y empleo. Nacional-Mujeres. Recuperado de: http://www3.inegi. org.mx/sistemas/tabuladosbasicos/tabtema.aspx?s=est\&c=33697

I INFOAGRO (2010). El papel de las mujeres en la agronomía. Ponencia. Recuperado de: http://www. infoagro.com/noticias/2010/5/16382_el_papel_mujeres_agronomia.asp 
I INMUJERES (2014). ¿Qué es Inmujeres? Historia. Recuperado de: http://www.inmujeres.gob.mx/ inmujeres/index.php/ique-es-el-inmujeres/historia

I Ito Sugiyama, E. y Vargas Núñez, B. (2005). Investigación cualitativa para psicólogos: de la idea al reporte. México: UNAM/Porrúa.

I Kvale, S. (2011). Las entrevistas en investigación cualitativa. España: Morata.

I Lamas, M. (2003). El género: la construcción cultural de la diferencia sexual. México: UNAM.

$\checkmark$ Licea de Arenas, J., Sandoval, M. y Arenas, M. (2003). La investigación agrícola en México. Un estudio bibliométrico con enfoque de género. Anales de Documentación, 6, 145-154.

I Miguez Fernández, P. (2007). El código de feminización y masculinización de las profesiones. México: UPN.

I Miranda López, F. (2004). La reforma de la política educativa: gestión y competencia institucional frente a la tradición corporativa. Sociológica, 19 (54), 77-123.

$\checkmark$ Razo Godínez, M. L. (2008). La inserción de las mujeres en las carreras de ingeniería y tecnología. Perfiles Educativos, 30(121), 63-96. Recuperado de: http://www.scielo.org.mx/scielo.php?script=sci arttext\&pid=S0185-26982008000300004\&lng=es\&tlng=es.

I Simón Rodríguez, E. (2006). Convivencia y relaciones desiguales. En C. Rodríguez Martínez (Comp.), Género y Currículo. Aportaciones del género al estudio y práctica del currículo. (pp. 153-167). Madrid: Akal.

I Sistema Nacional de Institutos Tecnológicos. Dirección General de Educación Superior Tecnológica (2012). Anuario Estadístico. México. Recuperado de: http://www.tecnm.mx/images/areas/planeacion/2012/ ANUARIO 2012 SNIT.pdf

I UAAAN (2012). Tercer informe de acciones y resultados. Recuperado de: https://administrativo.uaaan. $\underline{\mathrm{mx} / \mathrm{sdesarrolloed/CENEVAL2012.pdf}}$

I UACh (2011). Anuario estadístico. Recuperado de: http://www.chapingo.mx/upom/descargas/ae/ anuario e 2011.pdf

I UAEMéx (2013). Plan de Desarrollo Institucional 2010-2014. Ciencias Agropecuarias. Toluca. Estado de México. Recuperado de: http://www.uaemex.mx/planeacion/InfBasCon/CienciasAgricolas/ PlandeDesarrollo/2010-2014/PD 10-14 FCA.pdf 
I UAM (2011a). Resultados de la encuesta aplicada a egresados de la licenciatura en agronomía. División de Ciencias Biológicas y de la Salud. Unidad Xochimilco. México. Recuperado de: http://www.uam.

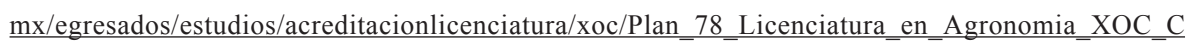
BS 2011 - copia.pdf

I UAM (2011b). Anuario estadístico. México. Recuperado de: http://www.uam.mx/transparencia/inforganos/ anuarios/anuario2011/anuario estadistico 2011.pdf

$\checkmark$ UNAM (2013). Agenda Estadística. Población Escolar. Licenciatura 2012.-2013. México. Recuperado de: http://www.planeacion.unam.mx/Agenda/2013/disco/xls/017.xls

I Vázquez García, V. y Zapata Martelo, E. (2005). Mujeres en universidades agronómicas y programas de estudios de la mujer en México y Estados Unidos. Un estudio comparativo. Revista de Estudios de Género. La Ventana, 252-280. Recuperado de: $\underline{\text { http://www.redalyc.org/articulo.oa?id=88402109 }}$

Envío a dictamen: 29 de enero de 2020

Reenvío: 28 de abril de 2020

Aprobación: 12 de mayo de 2020

Jorge García Villanueva. Psicólogo, Doctor en Psicología por la Universidad Nacional Autónoma de México (UNAM). Profesor titular en la Universidad Pedagógica Nacional (UPN) donde actualmente coordina el posgrado de Género en Educación. Su publicación más reciente es el libro La identidad masculina en jóvenes: una mirada, México, UPN, 2017, 172 pp. (Versión gratuita en inglés descargable: A Look into Masculine Identity in Mexican Young Men). Correo:jvillanueva@upn.mx

José Zenón Escobar Pérez. Licenciado en Planificación para el Desarrollo Agropecuario. Doctor en Liderazgo y Dirección en Instituciones de Educación Superior de la Universidad Anáhuac. Profesor titular de la Dirección General de Educación Tecnológica Agropecuaria, México. Correo: zenon_degeta@hotmail.com 
Claudia Ivonee Hernández Ramírez. Maestra en Desarrollo Educativo, Especialista en Género en Educación y Licenciada en Psicología Educativa por la UPN. Actualmente, trabaja en una investigación que está focalizada en la salud sexual y reproductiva desde la perspectiva de la sexualidad integral, en el campo de la Didáctica de las Ciencias y los Estudios de Género. Colabora como asistente de investigación en temas de género en educación en su Alma Mater. Correo: cihernandez@upn.mx 